\title{
Facilitating land use forecasting in planning agencies
}

\author{
G. Pozoukidou \\ Department of Planning \& Regional Development, \\ University of Thessaly, Greece
}

\begin{abstract}
History and current circumstances suggest that regional planning organizations will be major actors in the planning arena over the coming decades. Amongst other responsibilities they are now accountable for transportation and land use planning decisions which requires assessing the impacts of their transportation policies on land use development. Hence, these agencies are mandated to use sophisticated information management tools and complex land use modeling methods. Existing planning methods have proved to be extremely complex for use by regional agencies' untrained staff, which results in the inability of planning organizations to systematically assess the implications of their planning decisions.
\end{abstract}

It has been proposed that Knowledge Based Systems (KBS) can be used as a tool to improve planning practice by allowing untrained agency personnel to make effective use of complex planning methods. In this paper, we explore the role of KBS in assisting agency staff who lack modeling experience to make effective use of a sophisticated land use model. The hypothesis is that KBS can help us increase the usability of urban models by replicating the decision making process performed by land use model experts; increased usability will, in turn, increase their applicability in planning practice. The Transportation Economic and Land Use model (TELUM) was the software developed to demonstrate how KBS can refine existing land use models and create easy and simple to use planning support systems. The research concluded that under certain conditions KBS could be used as a method to create a buffer between the complexity of a land use modeling system and the inexperienced user.

Keywords: urban models, land use modelling, planning support systems, transportation and land use planning, knowledge based systems. 


\section{Introduction}

Both history and current circumstances suggests that regional planning organizations will be major actors in the U.S. planning arena over the next decades. With the enactment of Intermodal Surface Transportation Efficiency Act and Transportation Equity Act for the $21^{\text {st }}$ Century, new requirements and responsibilities were brought to elected officials regarding transportation decision-making. Metropolitan Planning Organizations (MPOs) and State Departments of Transportation (SDOTs) are responsible for transportation and land use planning decisions and are required to assess the impact of their transportation policies on land use development. Hence, these agencies are now mandated to use sophisticated information management tools and complex land use modelling methods.

A literature review on computer use in planning suggests that there is a continuous failure to use complex planning methods, like urban models, and decision support tools in planning practice [1]. There are several indications that their limited use and applicability in practice is due to their complexity. As a result there is an inability of planning agencies to systematically assess the implications of their planning decisions and thus fulfil their role. However, if there was a way to make models simpler and easier, to use and comprehend, then agencies may be able to effectively utilize them in everyday planning practices. This led to a search for appropriate technology that could help us achieve the goal of making complex systems like urban models, more usable and applicable to current planning practice.

The research was focused on how technology could help us increase the usability of urban models by replicating the decision making process performed by land use model experts. Knowledge Based Systems (KBS) have been proposed as an appropriate form of computer technology that could help us refine existing urban models and create more user-friendly planning support tools. Within this context a research hypothesis was formed as follows: Knowledge Based Systems can help us increase the usability of urban models by replicating the decision making process performed by land use model experts. Increased usability of urban models will in turn increase their applicability in planning practice. TELUM land use model was used to demonstrate how KBS can be used to refine existing land use models and create easy and simple to use planning support systems.

\section{TELUM case study}

TELUM, acronym for Transportation Economic and Land Use Model, is a land use modelling decision support system. Development of TELUM, which is part of a larger decision support system, was initiated and funded by the U.S. Department of Transportation, with Rutgers University and North Jersey Planning Authority being responsible for designing and developing the system. Funding started in 1998 with \$1 million per year, over six years period. In 2005 new funding was approved focusing on the implementation and widespread 
adoption of the system. The system was copyrighted and every MPO (350 in total) is eligible to use TELUM at no cost [2].

TELUM was designed with the intension to be an integrated interactive system that would assist MPOs to assess the land use effects of their transportation policies and choices. More specifically TELUM projects the location of future residential and employment growth based upon its analysis of the existing residential and employment location, land use patterns, location of the transportation improvement(s), and the overall congestion in the transportation system.

\subsection{TELUM structure}

In order for the reader to understand the complexity that the process of land use modeling entails, Figure 1 portrays in a very general way the interactions and interrelations amongst four basic agents: households, employers, developers, and government. Its purpose is to provide a structured way for the reader to think about land use modelling and to realize the implications, consequences, and reactions of any agent's actions. It also shows how land use modelling can be used to evaluate alternative governmental policies and investments.

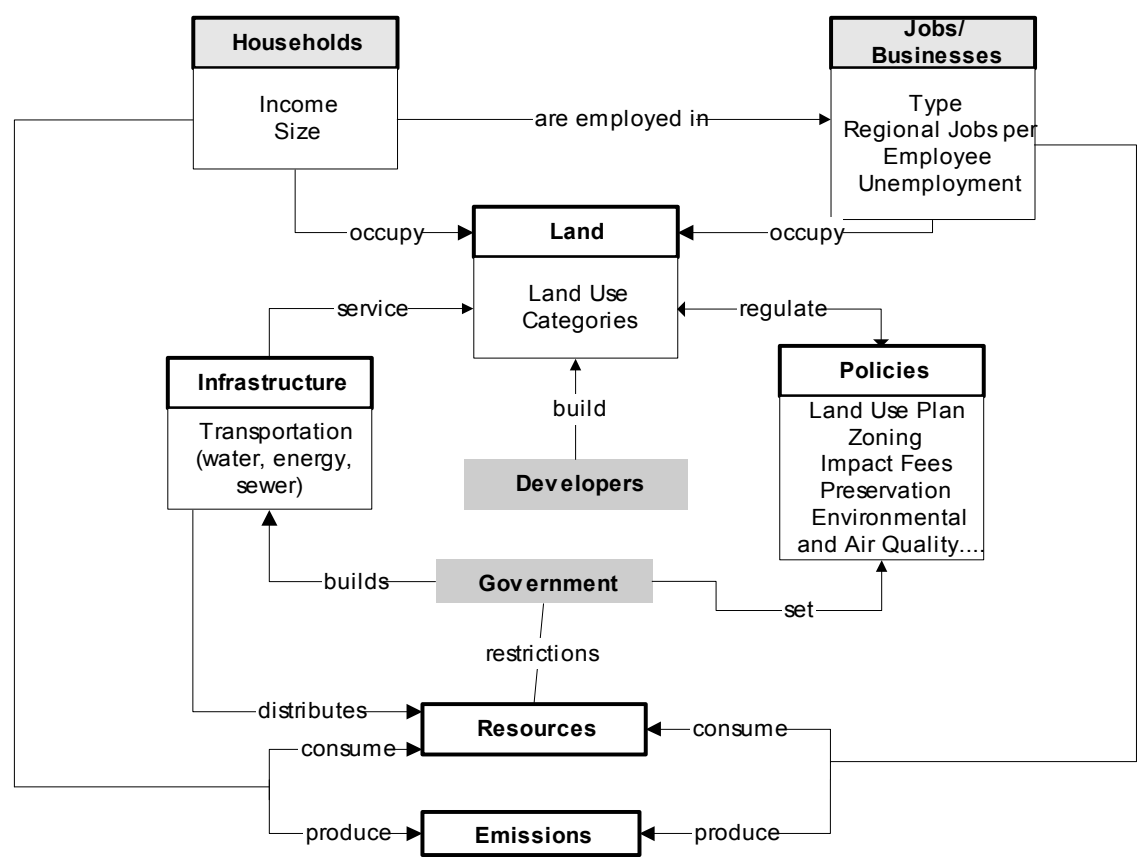

Figure 1: Interactions represented in TELUM.

Having this process in mind, TELUM attempts to replicate agents' interactions and record their consequences in a systematic way that will be easy for the user to comprehend. TELUM does not explicitly model every interaction amongst those agents, but it views them from an aggregative perspective. 
It focuses on how employment and their location choices affect the future location of the households, and in turn, the implications to the land use development patterns in the region. Actually, developers and government are more of exogenous factors to TELUM; nevertheless, they are important and should not be ignored since the main objective of modelling is to eventually do policy analysis.

The general configuration and structure of TELUM is shown in Figure 2, and consists of four main components. The first component is the Model Module which is associated with the computational utilities of the modelling process. The second is the Database Management System component, which is associated with preparing the data for analysis and display in various stages of the modelling process within the system. The third is the Geographical Information Systems (GIS) component, which is comprised of a GIS package that provides the user with visualization aids in various analysis phases. Finally, there is the Knowledge Based Systems, which is not a component of TELUM, per se, but is there to support the function of the three distinct modules and to communicate with the user.

The most important and probably most complex module is the Model module. The Model module is a collection of urban models that are necessary for the basic planning activities of analysis, prediction, prescription and evaluation that a planning agency performs. It is the place in TELUM where the actual modelling process is taking place. It consists of two major models: TELUMEMP and TELUM-RES. TELUM-EMP, the Employment Allocation Model, locates employers/employees. TELUM-RES, the Residential Allocation Model locates households to place of residence. There is also an internal sub model, the Land Consumption model, LANCON, which calculates the land consumption consequences of the previously calculated employment and household location demand.

In order for these models to perform their task, they have to employ two major computational utilities. The first is TELUM-CALIB, a calibration process, which uses a modified gradient search technique to calculate TELUM-RES and TELUM-EMP parameters, or coefficients, that produce the best fit for the model equations to region's data. The second computational utility is TELUMATRMOD, the attractiveness modification program, which is used to prepare the residual variation from the calibration results for use in the subsequent forecasting procedures and to provide a systematic procedure for incorporating exogenous information from local planners.

\subsection{The use of KBS in TELUM}

The structural analysis made obvious that each module is essential to the modelling process and that functionally they can be quite complicated. This exact fact, the complexity of the modelling task that is reflected in the structure and function of such systems, discourages the use of models especially when it comes to novice users. This is the part where Knowledge Based Systems (KBS) are found to be extremely useful. They could be used to hide the complexity of he land use modelling task and virtually perform the task for the user. 


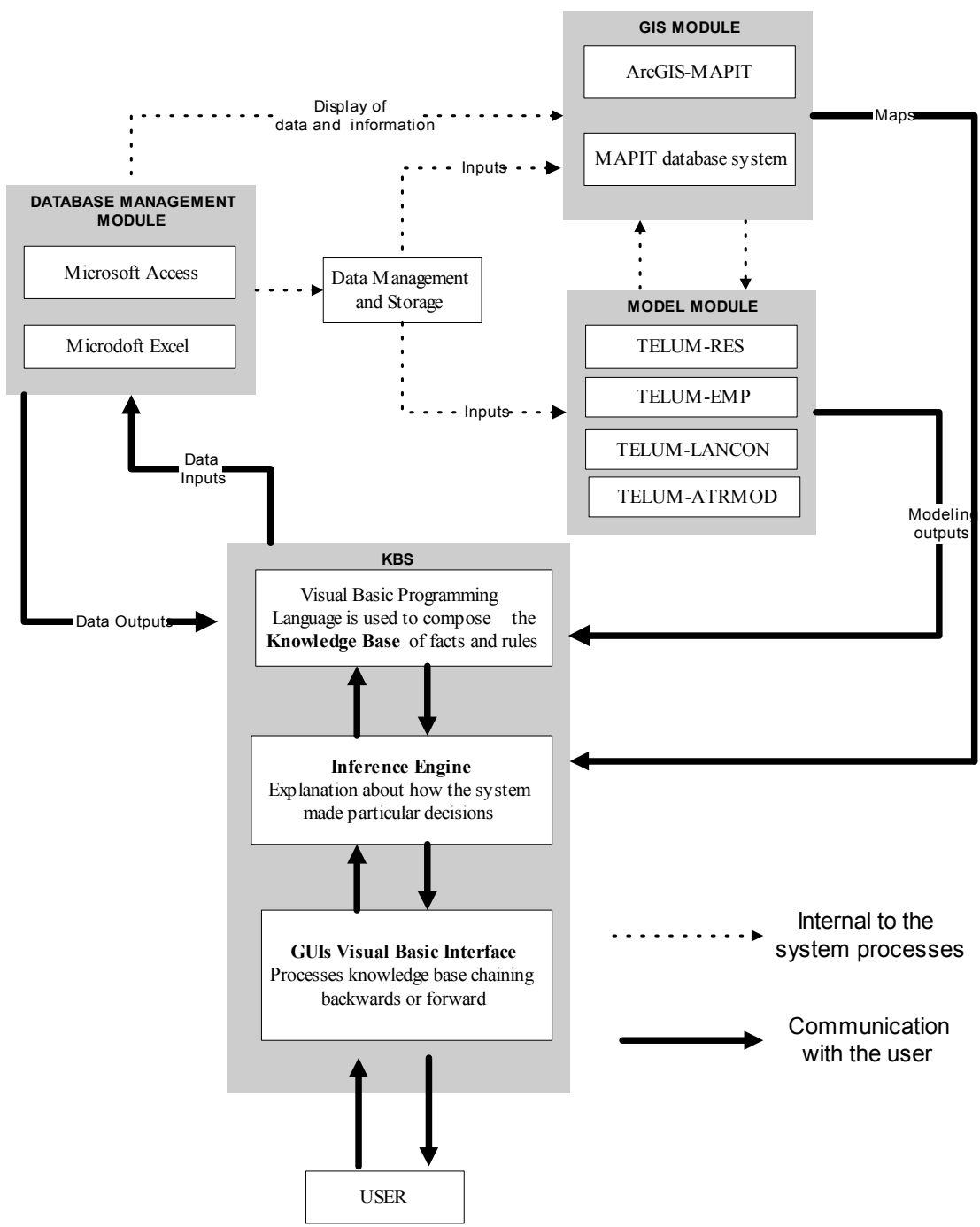

Figure 2: TELUM structural overview.

Knowledge Based Systems (K.B.S.) are computer programs that apply notions of artificial intelligence to narrow and clearly defined problems (Ortonalo and Perman, [4]). They are named for their essential characteristic, which is to provide advice in problem solving, based on the system's encoded knowledge from experts (land use experts in this case). The rationality behind this is that experts usually combine rules with facts to draw conclusions; a process that relies heavily on theories of logical deduction developed by mathematicians and philosophers, but are adapted to particular applications in a wide range of disciplines by engineers, scientists, and planners. 
Within land use modelling process KBS can be used as a way to interpret results or inferring situation descriptions from available data, diagnose malfunctions and prescribe remedies, support mathematical parts of modelling, like calibration, monitor and control expected outcomes, and to instruct specific tasks to the user. All these functions are performed with the help of three distinct parts of a KBS: the knowledge base, the inference engine, and the explanatory interface shown in Figure 2.

Figure 3 portrays a typical TELUM screen and provides an example of a very simple KBS function; diagnosing a potential malfunction and prescribing a remedy. The system asks the user to input data in this case, the number of zones and population size of the region being modelled. When the user clicks the "Continue" button the system automatically calculates the population per zone ratio. If the population per zone ratio does not satisfy certain criteria encoded in the knowledge base of TELUM, then the system warns the users about possible erroneous data inputs and makes "recovery" suggestions. The user can only see the front end of the function that KBS performs. Behind the scenes, there is a Visual Basic code that is "running the show".

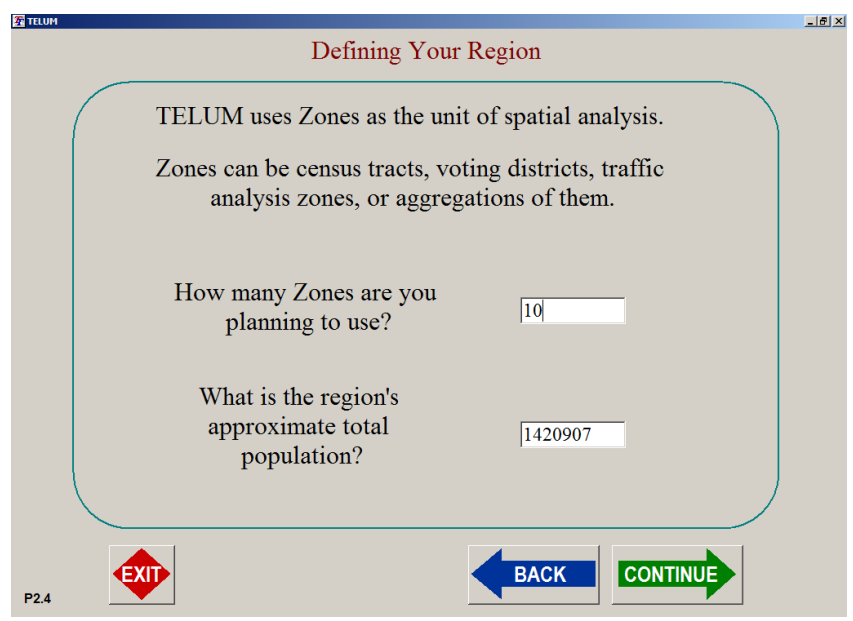

Figure 3: TELUM screen caption.

Under the same notion a whole set of rules were created in the form of $I F$ (condition true) THEN (action) and/or ELSE (action) and incorporated into the system in the form of Visual Basic code. As such, we created the knowledge base for TELUM and recorded expert's knowledge and experience regarding land use modelling. The creation of IF-THEN-ELSE rules may seem very simple concept for performing complex functions like this of modelling; nevertheless, one can imagine that a number of rules interconnected create a sophisticated system of accumulated knowledge.

In this context KBS have been used in TELUM as the vehicle to execute parts of land use modelling task on behalf of the user, hide the complexity and operationalize land use modelling task, monitor land use modelling events and 
procedures on behalf of the user, and finally train and teach novice users to perform the land use modelling task. They have also been used as a way to tie, relate and link different software packages like Microsoft Excel, ArcGIS, and the employment and household allocation models. Furthermore, they were used to link the different kinds of databases and forms of data that each one of the modules produces.

In order to perform these functions a set of more than 500 interconnected message frames or templates were developed. These message frames were used as a way to communicate between the user and the system, to input data into the system and to present outputs.

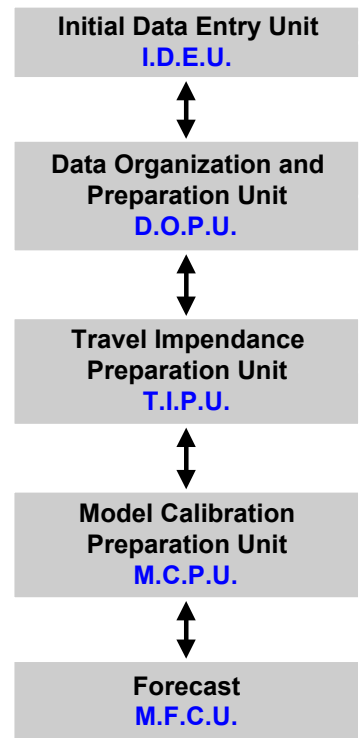

Figure 4: TELUM functional flow.

\subsection{Forecasting with TELUM}

To complete a full set of forecasts with TELUM the user has to go through five distinct sections. Figure 4 gives us an overview of the flow of the system. The user has to go through all sections in the order shown to complete a forecast. When the user enters TELUM for the very first time, s/he is required to go through the Initial Data Entry Unit (IDEU). The purpose of the IDEU section is to inform the user about the type of data that are necessary to perform forecasting. Data requirements can include a variety of spatial, temporal and sectoral dimensions. Furthermore, this section aims to help prepare and organize the required - for the modelling - data. This is just a preliminary step before entering real data into the system. Data Organization and Preparation Unit (DOPU), is where the data organization, data integration, data composition, and data consistency check is taking place. As soon as the user is in DOPU and 
Microsoft Excel has been activated, then the user has access to various spreadsheets that are tailored to the number of zones, number of employment and household categories and other type of data defined earlier in IDEU. By the end of the DOPU section the user has inputted almost all data needed for the modelling process. The only data missing are related to the transportation network of the region. TELUM itself does not include a travel demand model. It requires that the user provide the data in the form of travel cost or travel time matrices. Nevertheless, TELUM helps the user to organize and set the files ready for use in the subsequent modelling steps. All this takes place in the Travel Impedance Processing Unit (TIPU). The next step is calibration (Model Calibration Processing Unit-MCPU) which is probably one of the most crucial and significant parts of modelling that can highly affect the quality and validity of the forecast results. At the same time, the process itself is analogous to a regression analysis and it is a mathematically and statistically complex procedure. During calibration a series of statistical indices are created that the user needs to comprehend in order to be able to evaluate the success, or not, of the calibration procedure. After calibration is done and the user is informed about how good the data fits the model; the system is ready to run forecasts. Within Model Forecast Calculation Unit (MFCU), the user(s) has the opportunity to run a baseline forecast and to create alternative development scenarios for their region incorporating, if desired, local knowledge. The forecasting process starts with a baseline forecast using data inputs (DOPU), travel data (TIPU), and a set of model parameters that have been generated in MCPU.

Furthermore TELUM allows the user to specify policy inputs and then generate and compare alternative scenarios. It also allows interactive testing of how different policy strategies play in achieving a particular vision or set of objectives that have been set by a community. Governmental agencies, like MPOs, influence the development process through a mixture of land use regulations and infrastructure provisions. These are combined such as to foster the development patterns that support the objectives and vision for an area i.e. sustainable development. With the help of TELUM, agencies have the opportunity to quantify particular visions and objectives by developing various scenarios. Scenarios consist of a combination of development policies that might include comprehensive plans, infrastructure plans, urban growth boundaries, development restrictions, environmentally sensitive policies, etc.

\section{TELUM usability testing}

\subsection{Research method}

In order to test the hypothesis stated in the introduction of this paper, an evaluation of TELUM, by its users, was performed. The evaluation explored issues related to how KBS are used to provide a buffer between a system's complexity and user; augment user skills; open the "black box" of models; and overcome potential bottlenecks in the implementation of complex systems in planning practice. 
Usability inspection is a generic name for a set of methods that are used to examine the usability related aspects of user interfaces, to find out whether a system meets its intended purposes. It finds usability problems system design and then uses the problems to make recommendations for fixing them, which improves the usability of the system.

Heuristic Evaluation was the inspection method used to examine usability related aspects of TELUM's interface and to find out whether the system meets its intended purposes. "Heuristic Evaluation" is performed by having each evaluator inspect the system alone. During an evaluation session, the evaluator goes through the system several times and inspects various elements. The inspection is conducted by comparing these elements to a list of recognized usability principles-the heuristics. Heuristics are general rules that describe common system properties. The output from the evaluation is a list of usability problems with references to those principles that were violated by the design according to the opinion of the evaluator. Following is a short list of the heuristics developed by Molich and Nielsen, used to evaluate TELUM. These are [3]: Visibility of system status, Match between system and the real world, User control and freedom, Consistency and standards, Help users recognize, diagnose, and recover from errors, Recognition rather than recall, Flexibility and efficiency of use, Aesthetic and minimalist design, Help and documentation, User skills, Pleasurable and respectful interaction with the user.

\subsection{Users' (testers') profile}

The user team that performed the evaluation consisted of 17 members in two distinct groups. Five professional urban planners, representing five different MPOs, was the first group and 12 second year Master City Planning candidates were the second group. It should be noted that the students participated in the research were attending the "Advanced Urban Modelling Seminar" as part of their colloquium at the University of Pennsylvania. Evaluators' computer and urban and transportation modelling competence was assessed through a questionnaire. This information was necessary to better understand and interpret their responses over the course of the evaluation.

It was not to out surprise that none of the five professional City Planners participating in the study used any type of land use models in their planning processes. When it comes to transportation models, GIS, and statistical packages, they were familiar with basic concepts. Actually, for the purposes of this research, the fact that the users do not have any experience using land use modelling tools worked to our advantage; because, TELUM is a software targeting planners with novice land use modelling skills.

The profile of the student group was somewhat different. All of the participants were familiar with the theoretical aspects of land use models. In contrast to the first group, in this one none of the participants had any experience using transportation models. The group seemed to be competent when it came to GIS and statistical skills. The students, in general, seemed more confident using computer tools and more technologically savvy than the professionals. 


\subsection{Results analysis}

The 17 participants experienced a total of 244 usability issues, of which 42 were unique. Because there is an overlapping of errors found by the users, it is imperative to count them just once in order to have a more realistic accounting of the problems. Table 1 summarizes the 244 issues identified based on the 11 different criteria (heuristic guidelines) presented in the questionnaire.

The number shown in the column "Total Issues" represents the usability problems identified in the specific category. The highest frequency of errors occurred in the category of "Help and Documentation" with 60 issues identified, which represents $25 \%$ of the total usability issues. Second, there were the categories of "User Control and Freedom" and "Help Users Recognize, Diagnose, and Recover from Errors", which each accounted for $27 \%$ of the total usability issues. A short analysis of the results for each one of these categories is provided below.

Table 1: $\quad$ Number of usability issues identified.

\begin{tabular}{|c|c|c|c|c|}
\hline Issue Categories & $\begin{array}{l}\text { Total } \\
\text { Issues }\end{array}$ & $\begin{array}{c}\% \\
\text { Total }\end{array}$ & $\begin{array}{l}\text { Unique } \\
\text { Issues }\end{array}$ & $\begin{array}{l}\text { \% Unique } \\
\text { Issues }\end{array}$ \\
\hline 1. Visibility of System Status & 12 & 4.92 & 2 & 4.76 \\
\hline $\begin{array}{l}\text { 2. Match between system } \& \text { the } \\
\text { real world }\end{array}$ & 13 & 5.33 & 2 & 4.76 \\
\hline 3. User Control \& freedom & 32 & 13.11 & 4 & 9.52 \\
\hline 4. Consistency \& Standards & 25 & 10.25 & 5 & 11.90 \\
\hline $\begin{array}{l}\text { 5. Help Users Recognize, } \\
\text { Diagnose \& Recover from Errors }\end{array}$ & 33 & 13.52 & 4 & 9.52 \\
\hline 6. Recognition rather than recall & 14 & 5.74 & 4 & 9.52 \\
\hline 7. Flexibility \& efficiency of use & 11 & 4.51 & 2 & 4.76 \\
\hline 8. Aesthetic \& Minimalist design & 14 & 5.74 & 4 & 9.52 \\
\hline 9. Help \& Documentation & 60 & 24.59 & 8 & 19.05 \\
\hline 10. Skills & 12 & 4.92 & 1 & 2.38 \\
\hline $\begin{array}{l}\text { 11.Pleasurable \& respectful } \\
\text { interaction with the user }\end{array}$ & 18 & 7.38 & 6 & 14.29 \\
\hline TOTAL & 244 & 100 & 42 & 100 \\
\hline
\end{tabular}

Help and Documentation. When using TELUM users could get help through two distinct ways: an extensive system of hyperlinks and a paper manual. Hyperlinks were not intended to replace the manual, but to complement it. Hyperlinks were the main way users accessed the help. A large percentage $(80 \%)$ of them indicated that they did use the manual in cases where the information provided through the hyperlinks was inadequate. Users reported that hyperlinks had sufficiently offered help in regard to procedural directions and description of various tasks. Nevertheless, a lot of them (58\%) thought that the provided help should be even more descriptive and procedural of the tasks that take place in 
TELUM. An even more significant percentage of users (70\%) thought that the help system should also be more interpretive, a feature they thought was underdeveloped in the help system.

User Control and Freedom. Some users (8 out of 17) thought that TELUM was marginally functional. It is very interesting to note that the perceived level of control and freedom that the users desire varies and depends on the familiarity of the user with the software and on their computer competence; the more competent the user becomes (using TELUM) the less structure or more freedom is desired.

Help Users Recognize, Diagnose, and Recover From Errors. In general, users seemed to be satisfied with the ability of TELUM to diagnose and report errors over the course of the modelling task. Nevertheless, users' reported (9 out of 17) that the system lacked the ability to offer sufficient recovery suggestions and information regarding the source and cause of error.

The issue of not providing adequate recovery suggestions is, related to the complexity of the land use modelling task, meaning that a problem can have multiple sources which make impossible to create a comprehensive and exhaustive list of possible problem sources. At the same time, the fact that users indicated this as a problem is related to their limited understanding of quantitative problems and statistical concepts, which is in turn connected to the much wider issue of inadequate education and training of planners.

Flexibility and efficiency of use. "Flexibility and efficiency" of use of the system is directly related to "User control and freedom". TELUM was designed to be as flexible as possible within the limitations of the linear and sequential nature of the land use modelling task, which by rule does not leave a lot of room for adaptation. Again the different skill level of users becomes an issue in respect to the desired level of flexibility of the system.

\section{Conclusions}

TELUM evaluation enabled us to make interesting conclusions in regard to KBS's ability, as a technology and information systems tool, to address the issue of high expertise and advanced skills that a user is required to have to use such systems. KBS managed to reduce the complexity and increase the understandability of urban models incorporated in TELUM. Furthermore given the complexity of the land use model, the fact that inexperienced users thought that they need little or no expertise to use the system suggests that KBS is an effective method in introducing complex tools to novice agency staff. Analysis of results enabled us to make some further recommendations regarding the issue of high expertise required to utilize such systems.

1. User Expertise and Flexibility of Use. A system's perceived level of flexibility is relevant to the level of expertise that the user possesses and his/her familiarity with the system. The more familiar the user becomes with the system the more flexibility or the less control -of the user by the system- is desired.

The fact that users of TELUM had varied skill levels created two groups of users with distinct perspectives in regard to the efficiency and flexibility of 
TELUM. Throughout the evaluation it has been obvious that the more skilled users required more flexibility and freedom within the system, in contrast with, the less skilled users that were satisfied with the existing structure of the system.

2. User Expertise and System Automation. The desire to explore how models function (open the black box of the models) is related to the modelling skills of the user; the higher the skills and competence of the user the higher the desire to learn about models.

TELUM was designed to address the needs of the unskilled users. Therefore, to make models usable and understandable to this group of users, automation of certain land use modelling routines was necessary. At the same time, the choice of automating certain routines generated feelings of distrust, loss of control, and dissatisfaction with the more skilled users. Therefore, "opening the back box" of urban models is a relative notion and depends on the skills of the targeted users/audience.

The different preferences of these two groups (the unskilled and skilled users) created certain dilemmas and considerations with regard to the design strategies and principles of such systems. Thus, when designing systems, like TELUM, it is important that the developer:

- considers alternative ways to provide more or less flexibility and adjust to users' skill levels;

- avoids automation tactics that could transform users to think-less system operators;

- $\quad$ is sensitive to the fact that complexity of land use modelling concepts and procedures could be discouraging and overwhelming for the user.

Finally developer's decision about how much flexibility, automation and control over the user's actions should be based on two criteria: the targeted audience and the complexity that the land use modelling task entails. For instance, in the case of TELUM the system was designed with reduced flexibility to ensure that all users would be able to use the system with the least possible fatal errors.

In order to address such operational and design issues, the developer should seek ways to overcome certain KBS operational limitations. The operational capabilities (and limitations) of a system that incorporates KBS are related to the way the system acquires and uses the knowledge that is stored in the system. Increasing system capabilities to better respond to complex planning problems requires continuously encoding more knowledge into the knowledge base.

Hence, developers should seek ways to automate knowledge acquisition techniques or incorporate routines of machine learning that will enable the expansion and constant building up of the knowledge database. This will result in a continuous development of system capabilities that would make it possible for the system to address new situations and adapt to users' demands and skills.

In conclusion, using KBS could increase the usability of land use models since they create a buffer between the complexity of a land use modelling system and the inexperienced user. KBS could also be used as a technique to open the black-box of land use models; making them understandable and accessible to the less knowledgeable planning staff. However, successful application of systems 
that encompass KBS depends on their ability to serve and satisfy the needs of users that might have various skill levels.

\section{References}

[1] Brail R., Klosterman R.: Planning Support Systems. Integrating geographical information systems models and visualization tools. ESRI, p.1-23, California (2001)

[2] S.H.Putman Associates, Inc.: A Land Use Model for TELUS: MPO Data Availability Survey, Report (2000)

[3] Nielsen J., Mack R. (eds): Usability Inspection Methods, John Wiley and Sons, Inc., New York, p.25-62 (1994)

[4] Ortolano L., Perman C.: A Planner's Introduction to Expert Systems. Computer Report, Journal of the American Planning Association, 53(1), 98-103, (1987) 